\title{
Materials for Implantable Electrodes
}

The diva's voice, sweet and clear, fills the concert hall. Thousands of ears scoop up the sound. In each ear, vibrations bombard the eardrum. Small bones in the middle ear relay the vibrations to a snail-shaped structure called the cochlea. Hair cells in the cochlea translate the vibrations in to electrical signals that pulse along the auditory nerve to the brain.

We hear.

Unless, that is, one of these delicate structures has been damaged. More than 200,000 people in the United States suffer from sensorineural deafness caused by damage to the cochlea. A few of them have begun to hear again, thanks to decades of work toward developing an artificial ear-a cochlear implant.

Cochlear prostheses, being developed by several groups of scientists around the world, use microphones to collect sounds, then convert the sounds to electrical signals and deliver those signals via implanted electrodes to the auditory nerve for transmission to the brain.

They are just one of many types of neural prostheses that offer hope to neurologically impaired people. Devices have been or are being developed to control urinary incontinence, stimulate bone growth, reduce chronic pain, prevent epileptic seizures, restore motor function in paralyzed people, correct scoliosis, control respiration, and restore visual perception.

Implantable electrodes have been in use for over 25 years, in the form of cardiac pacemakers, which deliver regular electrical stimuli to hearts that have lost the ability to beat rhythmically on their own. Other types of devices work on a neural control principle, that is, they respond to directions given consciously by the user.

Like other types of prostheses, these devices must be manufactured from biocompatible materials. They must be noninflammatory, non toxic, nonallergenic, noncarcinogenic, and nonthrombogenic (not inducive of blood clots.) They also must be mechanically durable and chemically stable.

But implantable electrodes pose additional problems for their engineers. Because nerves lack the capacity to regenerate, any damage done upon insertion or while the implant is in place can leave the patient in worse condition than he began. This problem is compounded by electrochemical reactions between the implanted electrode and tissue. The high salinity of bodily fluids can lead to corrosion of the electrode, failure of the device, and release of toxic substances into the surrounding tissue. The neural prosthesis must be sufficiently non reactive that surrounding neural tissue remains both physically and physiologically intact.
Corrosion can also lead to chronic electrical stimulation of adjacent neurons and tissues with associated undesirable side effects. These include increased metabolic activity, vasoconstriction, formation of calcium deposits, epileptic seizures, and breakdown of the blood-brain barrier which normally protects the brain from circulating microorganisms and toxic substances.

\section{Other Problems to Overcome}

Damage to the tissue and damage to the electrode are not the only potential problems. In addition, the electrodes must not induce the body to respond with one of its traditional defense mechanisms-encapsulation. Normally the human body shields itself from foreign bodies by encapsulating them in connective tissue. However, this can render an implantable electrode nonfunctional by blocking access to the appropriate nerve.

Different types of neural prostheses, with their different functional and physiological requirements, demand different mechanical and electrical solutions to these problems.

For simpler types of implantable electrodes, the so-called single-channel devices, the pacemaker industry has developed reliable materials and manufacturing procedures. These devices-cardiac pacemakers, bone growth stimulators, some muscle stimulators and some pain reduction devices-generally use large-surface-area, low-impedance electrodes.

The more complex prostheses-multichannel devices that restore auditory or visual perception, or functional neuromuscular stimulation (FNS) devices that must incorporate sensory as well as motor nerve function-require tiny, precisely located and high-impedance stimulation contracts

Such devices place more stringent demands on the materials used. Insulation of the electrode becomes increasingly important; a poorly insulated electrode will not only fail to deliver sufficient current but in addition, places the surrounding tissue at greater risk. Insertion of the electrode and prevention of breakage also become more troublesome as the electrodes become smaller. And the higher currents necessary as a result of smaller surface areas mean increased risk of electrochemical corrosion.

\section{Biocompatibility}

Scientists at EIC Laboratories in Norwood, Massachusettes, led by S. Barry Brummer, have conducted a series of experiments on the electrolysis of various metals in a saline environment. They concluded that the noble metals platinum and iridium can safely induce ionic currents in body fluids at specifically designated charge densities. Platinum or platinum- iridium alloys have since been generally accepted as optimal electrode materials. Newer electrodes using iridium oxide surfaces are still in the research phase.

Given the parameters defined by Brummer et al., scientists still had to determine whether current levels which don't induce electrolysis are sufficiently low to prevent the problems of chronic electrical stimulation.

"We've been able to define safe levels using accelerated tests in animals," says F. Terry Hambrecht, head of the Neural Prosthesis Program, National Institute of Neurological and Communicative Disorders and Stroke, NIH. These tests can be extrapolated to study the decades in which an implantable electrode might be in place.

Insulating the electrodes to prevent electrical failure and current leakage, and to prevent electrical stimulation to tissue other than that intended has been a more vexing problem.

"The reason we have problems is, in part, because we have to use biocompatible materials," says Robert White of Stanford University. "Biocompatible materials are chemically inert, so if we want to make a laminated structure using an inert polymer with an inert metal on top of it as our conductor, the two don't want to stick together."

White's group at Stanford is one of at least nine groups around the world developing cochlear prostheses. Some simple, single-channel devices have been in use for as long as 25 years. Among the more complex multichannel devices that offer increasingly realistic sound, several approaches have proved successful.

\section{Development of Cochlear Implants}

A glimpse at how some scientists have attacked the problems of cochlear implants illustrates the complexity encountered in developing neural prostheses in general.

A group at University of California, San Francisco, headed by Michael Merzenich is testing an eight-channel device in nearly 20 patients. With Gerald Loeb of NIH, they settled on a platinum-iridium electrode coated with the vapor-deposited polymer. Parylene C (Union Carbide). Since Parylene doesn't bind to metal, an organic layer of polyimide Pyre-ML is deposited prior to Parylene coating.

Parylene- $C$ has been used increasingly in medical devices in recent years. In addition to pin-hole free insulation, it offers high resistivity and excellent biocompatibility.

The leads of UCSF's cochlear implant coil in to the cochlea, with eight bipolar stimulation contacts placed at precisely determined locations. In order to insert the

continued 
electrode, however, the fragile basilar membrane which separates two of the cochlea's three spiral canals, must be protected. Simply pushing a flexible electrode into the canal can be too damaging.

The UCSF team solved this problem by constructing their electrode from a stack of 16 Flattened wires (8 pairs). This "rib" resists bending out of the horizontal plane, but flexes easily into a spiral. Finally, the rib is molded into a Silastic (Dow) carrier which gives the implant elasticity and "memory" of the carefully determined spiral configuration.

The Stanford implant avoids the problem of potential damage to the basilar membrane altogether. Rather than coiling the electrode in to the cochlea and stimulating nerve endings close to the basilar membrane, a rigid tantalum on sapphire electrode array impales the auditory nerve and stimulates it directly.

White, chairman of the department of electrical engineering at Stanford, says his approach-thin-film planar photolithography-takes advantage of the techniques developed for integrated circuit production.

"What we're trying to do is use the technologies developed in the semiconductor industry to make small structures that you can fabricate in a batch fabrication mode with electrical and mechanical reproducibility from batch to batch as well as dimensional stability. We can work at very small dimensions which you can't do with bent wire technology."

In order to use photolithographic processes. White needed to find a suitable substrate that met three conditions: it had to be strong, a poor conductor of electricity so that elements on the array could be isolated, and biocompatible. Sapphire $\left(\mathrm{Al}_{2} \mathrm{O}_{3}\right)$ filled the bill. Platinum was chosen as the electrode metal. Only the tips of the electrode leads are platinum, however. The conductor is made from tantalum, which adheres readily to sapphire and which, by an anodically formed tantalum pentoxide $\left(\mathrm{Ta}_{2} \mathrm{O}_{5}\right)$ layer is well insulated against current leakage.

The array is further insulated from the saline environment by an overcoating of Parylene-C.

White's group is also developing a photolithographic, flexible array for insertion in to the cochlea.

All these implants have evolved over the years. The UCSF device, in a simpler form, has beenfunctioning in patients since 1972 . In the present configuration, UCSF prostheses have been in place for a maximum of 15 months.

"Three things are special about a cochlear implant," says Merzenich. "First is the required longevity. The device may be implanted in a young individual, therefore we want a 70 to 80 year lifespan. Second is the complexity of the high impedance electrode array. And third, you never want to disturb or replace the electronic components."

Merzenich says that as the electrode technology progresses and as scientists further elucidate how the ear processes information, cochlear prostheses will have the ability to recover full speech comprehension in children. But as a child's head grows, the device must maintain its integrity and its ability to function. If any damage occurs to the cochlea, the possibility of restoring auditory perception diminishes.

The general strategy developed at UCSF will be applicable to other types of complex stimulating neural prostheses, such as multiple-site muscle or bladder stimulating devices, adds Merzenich.

\section{The Future}

Photolithographic technology may hold even more promise for neural prostheses. The dimensions of electrodes fabricated this way are small-on the order of the the size of neurons themselves.

"We're working in the direction of smaller and more numerous electrodes for more complex stimulation patterns," says White. These will be especially useful for the auditory prostheses, which are perhaps the most complicated. Other possible uses include cortical stimulation for the control of spasticity and neuromuscular devices which must detect nervous impulses as well as stimulate individual nerves.

Extremely tiny electrodes are needed for such devices as well as complicated processing techniques which require implantable microelectronic chips. These chips need very thin insulation, notes Hambrecht.

The flexible electrodes will be especially useful for situations where the nerve must be wrapped in an electrode array, such as for bladder control or phrenic nerve stimulation devices (lung pacemakers.) Flexible electrodes may also be used in muscular stimulation, where the electrode and lead must flex with the muscle itself.

Also on the horizon are capacitor electrodes which have a thin layer of oxide right over the electrode surface. These electrodes inject charge by a capacitive process called double-layer charging in which neurons are stimulated with a voltage charge rather than by electron transfer. Thus there are no potentially dangerous electrochemical reactions.

"We can't make them small enough with sufficient charge densities," says Hambrecht. "There might be a fundamental limitation to that. But we still use them in research when we need a super-safe electrode."

While capacitor electrodes may not fulfill the promise once imagined, the oxide technology may provide answers to another problem-that of insulation. According to White and others, only a true chemical bond such as that between a metal and its oxide can effectively protect an electrode in a saline environment for the decades in which the electrode might be in place.

None of these solutions will be useful in all types of neural prosthesis however. "It's going to have to be very device-oriented," says Lois Robblee of ElC. For example, materials such as platinum-iridium while suitable for cochlear prostheses lack the mechanical qualities necessary for intramuscular electrodes. "As electrodes get smaller and smaller for single cell stimulation, that is, one nerve stimulated by one electrode, then we will need to have materials of very high charge capacity," she adds.

The requirements forstimulation charge density, current density and waveforms are so varied, she says, that many different electrode types will be useful.

"All of them have their own little niche."

L. J. BAIN

\section{Just Published by MRS}

\section{COMPUTER-BASED MICROSCOPIC DESCRIPTION OF THE STRUCTURE AYD PROPERTIES OF MATERIALS}

\author{
Volume 63 of the MRS Symposia Proceedings series \\ Edited by J. Broughton, W. Krakow, and S.T. Pantelides
}

Thirty-six papers comprise the proceedings of the symposium held at the 1985 MRS Fall Meeting. This first symposium of its kind for MRS served as a forum for the discussion of recent progress in microscopic descriptions of static and dynamic properties of materials by largescale computation.

MRS Members: $\$ 35$ US Nonmembers: $\$ 44$ Foreign Nonmembers: $\$ 49$

Order from Publications Department, Materials Research Society. 9800 McKnight Road, Suite 327, Pittsburgh, PA 15237; telephone (412) 367-3012.

Prepayment required. Mastercard and VISA accepted. 


\title{
BIOMEDICAL MATERIALS
}

\author{
Volume 55 \\ Edited by J.M. Williams, M.F. Nichols, and W. Zingg
}

New materials processing techniques and methods of analysis have equipped the materials scientist/ engineer as never before to respond to the unique challenges posed by in-vitro applications of materials. The first MRS symposium on biomedical materials brought together an international interdisciplinary group of experts to discuss developments in materials affecting the broad range of bioengineering practices around the world. The meeting emphasized developments in orthopaedics, electronic materials, and basic science and technology of biomaterials.

The proceedings contains 43 papers contibuted by scientists from the United States, Canada, China, France, Germany, Sweden, Switzerland, and UK. Topics include:

\section{Orthopaedics}

Ultrahigh molecular weight polyethylene in joint compounds - cemented UHMWPE total hip acetabular cups • use of $\mathrm{BMP}$ and cancellous graft for porous surfaced interface voids - $\mathrm{Al}_{2} \mathrm{O}_{3}$ fatigue fracture of ceramic-to-ceramic total hip replacements - improved ion implanted Ti-6Al-4V alloy for implants - nitrogen ion-implantation on the abrasive wear resistance of Ti-6Al-4V/UHMWPE couple

\section{Biomaterials for Electrodes}

Bioelectrodes for neuroprostheses - conductive polymer electrodes for neuroprotheses - stimulated electrodes based on $\mathrm{MnO}_{2}$ thin films - insulating polyphenylene oxide films on platinum electrodes - electronic materials for biological application

\section{Science and Technology of Biomaterials}

Chemical composition and morphology of titanium surface oxides • semi-solid processing of titanium alloys - fracture toughness testing of in-vitro aged biomaterials - microstructure, wear and friction of ion beam-induced amorphous Ti/Pd alloys - surface spectroscopic studies of solution cast biomedical grade polymer mixtures

\section{Cardiovascular Materials}

Small diameter vascular graft - failure of arterial prostheses - blood compatibility problems - calcium and magnesium with HEMA/MAA

\section{Opthalmic Biomaterials}

Biomaterials in opthalmology: a generalist's overview - materials for retinal detachment

\section{Reconstuction Materials for Bones and Teeth}

Composite plaster/hydroxylapatite intraosseous implants - slow crack growth in dental composites • reconstructive surgery of craniomaxillofacial deformation with osteomesh

\section{Novel Materials and Techniques}

Artificially induced biological organs - charged polymers as osmotic agents for peritoneal dialysis - spectroscopic ellipsometry studies of Giaever immunology slides - light and scanning electron microscopy for human spinal ligaments

\section{Simulation and Practice}

Plasma proteins in corrosion of $316 \mathrm{~L}$ stainless steel in vitro - stress corrosion cracking of stainless steel threaded fixation (Steinmann) pins for vertebral body stabilization - capsular contracture around PDMS and PE implants

$$
\begin{array}{ll} 
& \text { Hardbound, illustrated } \quad \text { ISBN: 0-931837-20-0 } \\
\text { MRS Members }-\$ 37.00 & \text { U.S. Nonmembers }-\$ 46.00 \quad \text { Foreign Nonmembers- } \$ 51.00
\end{array}
$$

Order from: Publications Department, Materials Research Society, 9800 McKnight Road, Suite 327, Pittsburgh, PA 15237; telephone (412) 367-3012

Prepayment required. VISA and Mastercard accepted. 